liegend, daß es sich bei diesen schwarzen Substanzen um Anlagerungsprodukte von Schwefel an $\mathrm{PbO}$ handelt, wobei man aus der Farbe und dem Auftreten ausgeprägter UltrarotPhotoeffekte den Schluß ziehen könnte, daß es sich um den höheren Bleioxyden analoge Substanzen mit teilweise vierwertigem Blei handeln dürfte. - Bemerkt sei noch, daß sich nach einem orientierenden Versuch auch bei der Einwirkung von aufgedampftem Selen auf eine dünne PbO-Schicht eine ganz analog aussehende dunkle Substanz bildete, die ebenfalls einen deutlichen Photoeffekt lieferte.

Zusammenfassend läßt sich also sagen, daß bei der üblichen, oxydierenden Sensibilisierung von Bleisulfid-Photozellen ${ }^{10}$ folgende Vorgänge eine wesentliche Rolle spielen können:

1. der Einbau von überschüssigem Schwefel in das PbS, der offenbar für das Auftreten träger Photoeffekte, besonders bei tieferen Temperaturen, verantwortlich sein dürfte,

2. die Einwirkung von Sauerstoff auf $\mathrm{PbS}$, die in erster Stufe zu PbO und Schwefel führt (vgl. ${ }^{1}$ ),

10 Es darf wohl angenommen werden, daß auch bei der oxydierenden Sensibilisierung von PbSe- und PbTe-Photozellen analoge Vorgänge stattfinden.

\section{Zur Theorie des Hall-Effektes ferromagnetischer Legierungen}

Von Klaus Meyer

Theoretisch-Physikalisches Institut der Universität Jena

(Z. Naturforschg. 10a, 656-657 [1955]; eingegangen am 20. Juni 1955)

Der Hall-Effekt der Ferromagnetika wird bekanntlich durch den Ausdruck

$$
E_{y}=\left(R_{0} H_{z}+R_{1} M_{z}\right) I_{x}
$$

beschrieben ${ }^{1} ; E_{y}$ ist die elektrische Feldstärke senkrecht zum Magnetfeld $H_{z}$ bzw. zur Magnetisierung $M_{z}$, diese drei GröBen stehen wiederum senkrecht zum elektrischen Strom mit der Stromdichte $I_{x}$.

Während die starke Temperaturabhängigkeit der außergewöhnlichen Hall-Konstanten $R_{1}$ mit der Theorie von $\mathrm{K}$ a r p l u s und L u t t in g e ${ }^{2}$ zu verstehen ist, wird das Verhalten der gewöhnlichen Hall-Konstanten $R_{0}$ im großen und ganzen durch die Theorie des Hall-Effekts der nichtferromagnetischen Metalle wiedergegeben. Bei ferromagnetischen Legierungen von Kupfer und Nickel hat jedoch $R_{0}$ eine schwache, aber genügend ausgeprägte Temperaturabhängigkeit: $\left|R_{0}\right|$ nimmt zu, wenn die absolute Temperatur $T$ abnimmt ${ }^{3}$. Dieses Verhalten ist mit Hilfe der Theorie der elektrischen Leit. fähigkeit der Ferromagnetika von $\mathrm{M} \mathrm{ot} \mathrm{t}{ }^{4} \mathrm{zu}$ deuten ${ }^{3}$. Hier soll darauf hingewiesen werden, daß sich, mindestens in einfachen Fällen, aus dem s-d-Austauschmodell von Won s o w s k i ${ }^{5}$ ähnliche Folgerungen ziehen lassen.

Die Voraussetzungen sind folgende: 1. Die Leitfähigkeit soll im wesentlichen durch $4 \mathrm{~s}$-Elektronen in einem fast leeren $4 \mathrm{~s}$-Band bedingt sein; das bedeutet eine Beschränkung auf

1 E. M. P u gh u. N. R o s to ker, Rev. Mod. Phys. 25, 151 [1953].

2 R. K a r plus u. J. M. L u t t ing e r, Phys. Rev. 95, 1154 [1954].

3 E. M. P u g h, Phys. Rev. 97, 647 [1955].
3. die Reaktion von $\mathrm{PbO}$ mit Schwefel beim Erhitzen, die bei rechtzeitigem Abbruch zu praktisch trägheitsfreien Ultraroteffekten bei Zimmertemperatur Veranlassung gibt, und

4. die Chemisorption von $\mathrm{O}_{2}$ an $\mathrm{PbO}$ bzw. der Einbau von Sauerstoff in das PbO-Gitter, die zu ebenfalls praktisch trägheitsfreien Photoeffekten bei Zimmertemperatur im sichtbaren Gebiet führen.

Durch Uberlagerung dieser Vorgänge und der dabei resultierenden Photoeffekte sowie durch das Entstehen dünner Deckschichten weiterer Oxydationsprodukte $\left(\mathrm{PbSO}_{4}\right.$, $\mathrm{PbO} \cdot \mathrm{PbSO}_{4}$, evtl. $\mathrm{PbO} \cdot \mathrm{PbS}_{2} \mathrm{O}_{3} \cdot \mathrm{x} \mathrm{H}_{2} \mathrm{O}$ und Bleicarbonate) lassen sich die in der Literatur beschriebenen, z. Tl. stark voneinander abweichenden lichtelektrischen Eigenschaften von PbS-Photozellen (wie spektrale Verteilung, Temperaturoptimum und Trägheit des Photoeffekts, Luftempfindlichkeit der Zelle usw.) wenigstens qualitativ weitgehend deuten, wie in einer folgenden Mitteilung gezeigt werden soll. Weitert Untersuchungen sind im Gange.

Die Durchführung der Untersuchungen wurde durch die Gewährung eines Forschungsstipendiums aus ERP-Mitteln ermöglicht. Der Gesellschaft von Freunden der Technischen Universität danken wir für eine Sachbeihilfe.

Legierungen, die noch nicht zu viel Nickel enthalten. 2. Wie üblich und abweichend von Wonsowski wird angenommen, daß der Beitrag der $4 \mathrm{~s}$-Elektronen zur spontanen Magnetisierung zu vernachlässigen ist.

Unter diesen Voraussetzungen genügt zur Behandlung des gewöhnlichen Hall-Effektes ein Zweibändermodell (je ein Unterband für $4 \mathrm{~s}$-Elektronen mit Plus- und Minusspin). Man erhält

$$
R_{0}=-\frac{1}{e n c} \frac{\sigma_{+}{ }^{2}+\sigma_{-}{ }^{2}}{\left(\sigma_{+}+\sigma_{-}\right)^{2}} ;
$$

$n$ bedeutet die Anzahl der 4 s-Elektronen in je einem Unterband,

$$
\sigma_{+}=e^{2} n \tau_{+} / m_{+}^{*}
$$

die Leitfähigkeit, $m_{+}{ }^{*}$ die effektive Masse am unteren Bandrand, $\tau_{+}{ }^{*}$ die Stoßzeit im Unterband mit Plus- bzw. Minusspin. Auf Grund der Blochschen Theorie der elektrischen Leit. fähigkeit der Metalle gilt in der Näherung der effektiven Masse

$$
\tau_{+} \sim 1 / T m_{+}^{*} .
$$

Der offen gelassene Faktor enthält nur noch temperaturunabhängige Größen. Mit den oben angegebenen Voraussetzungen erhält man aus der Theorie von Wonsowski

$$
m_{+}{ }^{*}=m_{0}^{*} /(1 \pm \lambda M) \text {; }
$$

$M$ ist die spontane Magnetisierung bei der Temperatur $T$, $\lambda$ eine Konstante, deren numerischer Wert allerdings höchstens indirekt durch Vergleich mit dem Experiment ermittelt werden kann ${ }^{6}$. Damit erhält man

$$
R_{0}=-\frac{1}{2 \text { enc }} \frac{1+6(\lambda M)^{2}+(\lambda M)^{4}}{\left[1+(\lambda M)^{2}\right]^{2}} .
$$

${ }^{4}$ N. F. M o t t , Proc. Roy. Soc. A 153, 699 [1936].

5 S. W. W o n s ow sk i, Zh. eksper. teor. Fiz. 11, 981 [1948].

${ }^{6}$ Dabei wird angenommen, da $\beta \lambda$ in dem betrachteten $B e$ reich wenig von der Zusammensetzung der Legierung abhängt. 
Das bedeutet folgendes: Bei fester Zusammensetzung der Legierung wächst der Betrag von $R_{0}$ mit wachsendem $M$, d. h. fallender Temperatur, $|\lambda M|<1$ vorausgesetzt. (Ist für alle Temperaturen $M=0$, d. h. die Legierung nicht ferromagnetisch, so ist $R_{0}$ temperaturunabhängig, soweit keine anderen Effekte mitspielen.) Dann wächst auch bei fester Temperatur der Betrag von $R_{0}$ beim Übergang von reinem Kupfer zu Nikkel-Kupferlegierungen mit wachsendem Nickelgehalt (abnehmendes $n$, zunehmendes $M$ ), bis die wachsende Löcherleitung im $3 \mathrm{~d}$-Band genügend groß geworden ist und damit die Grenze des Anwendungsbereichs der Theorie erreicht ist. Qualitativ entsprechen diese Folgerungen den Beobachtungen.

7 Dabei wird angenommen, da $\lambda$ in dem betrachteten Bereich wenig von der Zusammensetzung der Legierung abhängt.
Es erscheint vernünftig anzunehmen, da $\beta|\lambda M|$ für alle Temperaturen näher an Null als bei Eins liegt, da es andernfalls nicht gerechtfertigt ist, den Beitrag der $4 \mathrm{~s}$-Elektronen zur spontanen Magnetisierung zu vernachlässigen. Andererseits lassen sich ganz analoge Resultate mit Hilfe der Theorie der Leitfähigkeit der Ferromagnetika von $\mathrm{Mot} \mathrm{t}^{4}$ erzielen, so daß etwa die Anomalie der elektrischen Leitfähigkeit sicher nicht allein auf die von Wonsowski angenommene Ursache zurückzuführen ist. Angesichts der Tatsache, daß die in die Theorie eingehenden Parameter nur sehr ungenau abgeschätzt werden können, kann man zunächst nur annehmen, daß beide Ursachen beim Zustandekommen der fraglichen Effekte beteiligt sind, abgesehen davon, daß auch noch die Näherung der temperaturabhängigen Eigenwerte, die Wonsowski macht, einer Rechtfertigung durch eine strengere Theorie bedarf.

\section{Das gyromagnetische Verhältnis des Protons}

Von F. Kirchner und W. Wilhel my

I. Physikalisches Institut der Universität Köln

(Z. Naturforschg. 10a, 657-658 [1955]; eingegangen am 26. Mai 1955)

Während die spezifische Ladung der Elementarteilchen, also das Verhältnis ihrer elektrischen Ladung zu ihrer Masse, schon in sehr zahlreichen Untersuchungen mit großer Genauigkeit gemessen worden ist, liegt für das Verhältnis des magnetischen Moments zum Drall, also für das gyromagnetische Verhältnis des Protons, bis heute erst eine einzige Präzisionsmessung vor, die von T h o m a s, D r i s c oll und Hipple im National Bureau of Standards durchgeführt worden ist ${ }^{1}$. Die Messung beruht auf der Anwendung des B l o c h - P u r c ell schen Resonanzverfahrens, das bekanntlich die Kreisfrequenz der Kreiselpräzession, die den Teilchen im Magnetfeld aufgezwungen wird, mit sehr großer Genauigkeit zu messen gestattet; das gyromagnetische Verhältnis erhält man dann als diejenige Konstante, mit der man die Feldstärke des Magnetfeldes multiplizieren muß, um die gemessene Kreisfrequenz zu erhalten. Die Feldstärke des von Thomas, Driscoll und Hipple verwendeten Magnetfeldes wurde mit einer Cotton-Waage gemessen; sie betrug ungefähr $5000 \mathrm{Gauß}$. Für das gyromagnetische Verhältnis des Protons ergaben diese Messungen das Resultat:

$$
\gamma=(2,67523 \pm 0,00006) \cdot 10^{4} \mathrm{sec}^{-1} \mathrm{Gau}^{-1},
$$

wobei die Fehlerangabe als wahrscheinlicher Fehler anzusehen ist ${ }^{2}$.

Wir haben ähnliche Messungen durchgeführt, wobei wir aber das Magnetfeld nicht mit einem Elektromagneten, sondern mit einer eisenfreien Spule erzeugt und seine Feldstärke aus dem Spulenstrom und den Abmessungen der Spule berechnet haben. Die von uns verwendete Feldstärke betrug etwa $100 \mathrm{Gauß}$. Die Achse der Spule wurde in die

1 H. A. Th o m a s, R. L. D r is c oll u. J. A. H ip ple, Phys. Rev. 75, 902 [1949]; 78, 339 und 787 [1950].

2 An den oben angegebenen Meßwerten ist noch eine diamagnetische Korrektur von $2,7 \cdot 10^{-5}$ anzubringen (vgl. z. B. N. F. R a m s a y, Phys. Rev. 78, 339 [1950]). Unser Wert erhöht sich dadurch auf
Richtung des Erdfeldes gelegt, so daß der Einfluß des Erdfeldes durch Umpolung des Spulenstromes eliminiert werden konnte.

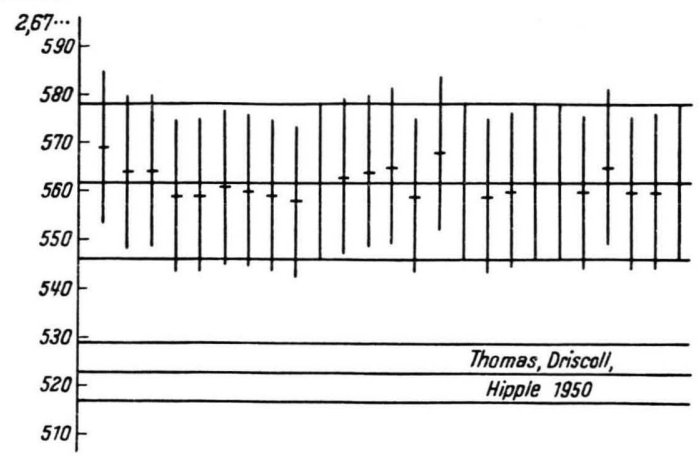

Abb. 1. Das gyromagnetische Verhältnis des Protons. Oben: eigene Messungsergebnisse. Unten: Ergebnis von $\mathrm{T} \mathrm{h}$ o m a s, $\mathrm{Dr}$ is coll und $\mathrm{Hipple}$.

(Die Differenzmessung liefert übrigens die Stärke des Erdfeldes auf ca. 1\%00 genau.) Unsere Messungen in der eisenfreien Spule liefern für das gyromagnetische Verhältnis des Protons

$$
\gamma=(2,67562 \pm 0,00016) \cdot 10^{4} \sec ^{-1} \mathrm{Gau}^{-1},
$$

wobei die Fehlerangabe den größtmöglichen Fehler darstellen soll ${ }^{2}$. Wie aus der graphischen Darstellung der Messungsergebnisse zu ersehen ist, besteht zwischen den Ergebnissen unserer Messungen und denjenigen von Thomas, Driscoll und Hipple eine Differenz, die weit größer ist als die beiderseitigen Meßfehlergrenzen. Da es wenig wahrscheinlich ist, daß das gyromagnetische Verhältnis des Protons von der magnetischen Feldstärke abhängt, und da man auch wohl kaum annehmen kann, daß sein Wert in einem Magnetfeld mit Eisenkern kleiner wäre als in einem eisen-

$$
\gamma=(2,67569 \pm 0,00017) \cdot 10^{4} \mathrm{sec}^{-1} \mathrm{Gau}^{-1},
$$

während Thomas, Driscoll und Hipple als diamagnetisch korrigierten Wert

$$
\gamma=(2,67530 \pm 0,00006) \cdot 10^{4} \mathrm{sec}^{-1} \mathrm{Gau}^{-1}
$$

angegeben haben. 